\title{
Case Report \\ Q Fever Presented as a Large Retroperitoneal Pseudotumoral Mass
}

\section{Behdokht Nowroozizadeh, ${ }^{1}$ Negar Haghighi Mehmandari, ${ }^{1}$ Nicolas Gallegos, ${ }^{1}$ Mari Perez-Rosendahl, ${ }^{2}$ and Di Lu ${ }^{1}$}

\author{
${ }^{1}$ Departments of Pathology, UC Irvine Medical Center, UC Irvine School of Medicine, Orange, CA, USA \\ ${ }^{2}$ Departments of Pathology, UCLA Medical Center, 10833 Le Conte Ave., Los Angeles, CA 90095, USA
}

Correspondence should be addressed to Di Lu; lud5@uci.edu

Received 2 August 2017; Revised 11 October 2017; Accepted 18 October 2017; Published 15 November 2017

Academic Editor: Hiroko Kuwabara

Copyright (C) 2017 Behdokht Nowroozizadeh et al. This is an open access article distributed under the Creative Commons Attribution License, which permits unrestricted use, distribution, and reproduction in any medium, provided the original work is properly cited.

\begin{abstract}
Background. Q fever is an infection caused by Coxiella burnetii, an intracellular organism. Acute infection is most often a benign and asymptomatic process; however, some individuals may go on to develop subacute and persistent localized symptomatic Q fever. As such, the clinical and histopathologic findings of $Q$ fever are widely variable and may be missed if clinical suspicion is not high. Case Presentation. Herein we report the first case of $C$. burnetii infection presenting as an isolated retroperitoneal mass. A 61-year-old male underwent axillary-bifemoral bypass surgery. His postoperative course was complicated by the discovery of a large retroperitoneal mass. Conclusion. Clinical and histopathologic findings of Coxiella burnetii infection are variable and can be deceiving. These are often nonspecific, especially in its persistent localized infectious stages.
\end{abstract}

\section{Introduction}

Q fever is a zoonotic infection caused by the obligate intracellular gram-negative pathogen Coxiella burnetii. Acute or persistent localized infection can occur, often with variable clinical and pathological features [1].

Acute $\mathrm{Q}$ fever infection is asymptomatic in $60 \%$ of cases but can manifest with nonspecific symptoms like fever, myalgia, malaise, hepatitis, and pneumonia [2]. Common manifestations of persistent localized Q fever infection include endocarditis, infectious thoracoabdominal aortic aneurysm, vascular graft infection, bone lesions, pulmonary infection, and granulomatous hepatitis. Encephalitis, pericarditis, and myocarditis have also been reported [3, 4]. In addition, there are rare reports of Coxiella burnetii infection presenting as mass lesions in the lung, mimicking malignant tumors radiographically $[5,6]$.

In our case, the patient presented with a large retroperitoneal mass that was clinically and radiographically suspicious for a sarcoma. CT-guided biopsy of the mass showed extensive ischemic-like necrosis involving skeletal muscle and fibroconnective tissue. Typical histologic characteristics of infection or inflammation were absent. Clinical work-up with serologic studies, however, revealed a high titer of $C$. burnetii antibody.

\section{Case Presentation}

A 61-year-old Vietnamese male with a past medical history of coronary artery disease, peripheral vascular disease, and abdominal aortic aneurysm underwent an axillary-bifemoral bypass and bilateral lower extremity thromboembolectomy. He was referred to our tertiary care center when his preoperative course was complicated by malaise, productive cough, and weight loss of 5 pounds over 5 weeks. The patient was a smoker and was currently employed as an electronics engineer. He denied night sweats, tuberculosis exposure, foreign travel, or animal contact in the past six months. He did report a cattle field along his route to work.

Abnormal laboratory values were as follows: erythrocyte sediment rate more than $120 \mathrm{~mm} / \mathrm{hr}$, white blood cell count of $18.8 \times 10^{9}$ per liter (L), $82 \%$ neutrophils, $12 \%$ lymphocytes, $6 \%$ monocytes, and hemoglobin of $8.5 \mathrm{~g} / \mathrm{dL}$. Computed 


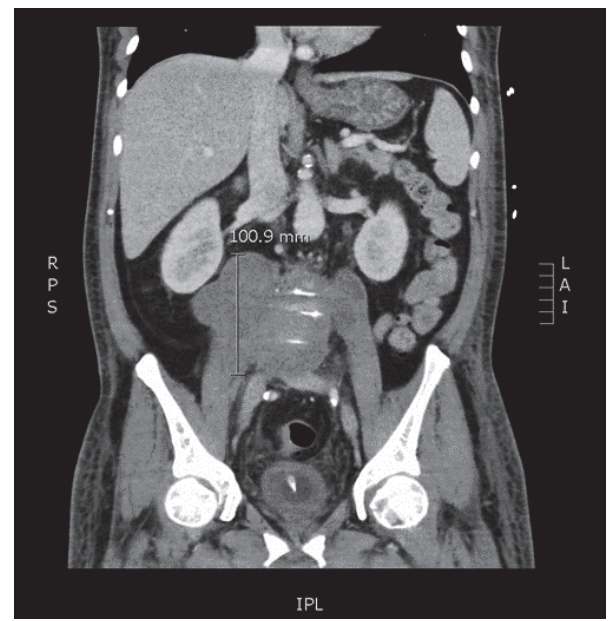

Figure 1: Abdominal CTA showing a lobulated low-density soft tissue mass within the retroperitoneum with involvement of the bilateral psoas muscles.

tomography (CT) scan of the chest showed bilateral ground glass opacities, most prominent in the right lower lobe, consistent with pulmonary edema. There was no evidence of a mass lesion in the chest. Abdominal computed tomography angiography $(\mathrm{CTA})$ showed a retroperitoneal $13.1 \times 10 \times$ $5 \mathrm{~cm}$ hypodense mass lesion at the L3-L5 level, extending to the bilateral psoas muscles (Figure 1). The mass was associated with bone destruction and sclerosis of the adjacent L4 vertebral body.

Magnetic resonance imaging of the abdomen confirmed a $13.9 \times 5.1 \times 10.3 \mathrm{~cm}$ lobulated retroperitoneal mass extending from the left to right psoas muscle. Possible invasion to the posterior wall of the infrarenal abdominal aorta and ventral L4 vertebral body was seen, raising concern for an infectious or neoplastic process. QuantiFERON-TB testing was negative, as was serologic testing for Treponema pallidum, Cryptococcus, Histoplasma, Coccidioides, and Brucella. Semiquantitative indirect immunofluorescent assay showed high anti-C. burnetii antibody titers: phase I IgG titer of $1: 16384$ with reference range of $<1: 16$ (performed by ARUP laboratories) and phase II IgG titer of 1: 4096 with reference range of $<1$ : 16 (performed by ARUP laboratories). CT-guided core biopsy was performed to evaluate the retroperitoneal mass, which revealed ischemic necrosis involving skeletal muscle and fibro connective tissue (Figure 2).

Acid-fast bacilli (AFB) and Gomori methenamine silver (GMS) stains were negative for acid-fast bacilli and fungal organisms. Immunohistochemistry for CD34, CD117, S100, and pankeratin was negative. Desmin and myogenin immunostains were positive in benign skeletal muscle.

Because there was a high index of suspicion for a malignant process, a second CT-guided core biopsy was performed one week later; histopathological findings were reminiscent of the first biopsy and showed no evidence of malignancy. At this point, the overall clinical and histopathological findings raised a high index of suspicion for C. burnetii infection. Tissue from the core biopsy was sent to the Health
Department of Orange County, California, where a direct PCR test for C. burnetii confirmed the diagnosis. The patient began treatment for $\mathrm{Q}$ fever with doxycycline $100 \mathrm{mg}$ twice a day and hydroxychloroquine $600 \mathrm{mg}$ daily. He showed marked clinical improvement with this treatment regime. Three months later, a repeat CT scan showed significant decrease in the size of the retroperitoneal mass, with maximum dimension decrease to $6.5 \mathrm{~cm}$, down from $13.9 \mathrm{~cm}$ at presentation. The patient recently completed an 18 month treatment course without complication. Final posttreatment imaging and serology are not available at our institution for review.

\section{Discussion}

Various case reports exist with respect to atypical presentations of $\mathrm{Q}$ fever. Q fever has reportedly been associated with abdominal aortic aneurysm, aortoenteric fistula, lower limb fistula, vascular graft infection, vertebral body erosions, CNS infection, fever of unknown origin, and possible pancreatitis $[3,4,7-10]$. Mass lesions are one of the rarest presentations of $\mathrm{Q}$ fever. To our knowledge, there have only been 2 previously reported cases, both lung masses [5, 6]. Janigan and Marrie reported a case, which presented with a single lung mass near the left para-aortic region, detected by chest X-ray and CT scan. A left upper lobectomy was performed. Histopathologic examination revealed small edematous bronchioles occluded by fibroblasts and sparse collagen intermixed with inflammatory cells. The diagnosis of $\mathrm{Q}$ fever was rendered after electron microscopy revealed the organism [5]. Lipton et al. presented a case of a right lower lobe lung mass with irregular borders seen on imaging. Bronchoscopy and fine needle aspiration failed to provide a definite diagnosis; the diagnosis of $C$. burnetii was made based on serologic markers [6].

Typically, the inflammatory/immune response to $\mathrm{Q}$ fever results in granulomatous lesions, most commonly seen in the lungs, liver, and bone marrow. The characteristic finding of $\mathrm{Q}$ fever is the so-called donut granuloma or fibrin ring granuloma, which consists of dense fibrin rings surrounding central lipid vacuoles. In persistent localized infection, pathologic findings may also include lymphocytic infiltration and foci of spotty necrosis [2].

To our knowledge, this is the first case reported of $\mathrm{Q}$ fever presenting as a solitary retroperitoneal mass. Due to the clinical suspicion for malignancy, CT-guided biopsy was performed twice. Histopathologic findings for both procedures revealed ischemic-like tissue necrosis without significant inflammation or granulomatous change. This is not typical of any infectious process but may be a unique finding for $\mathrm{Q}$ fever. Although the patient has no apparent direct exposure to animals, the cattle field located on his way to work could be regarded as an indirect exposure. In our case, the suspicion for $\mathrm{Q}$ fever was raised by the elevated titer, and the diagnosis was confirmed by PCR performed on the biopsy tissue. The clinical response to antibiotic therapy further confirmed the infectious nature of the mass. 

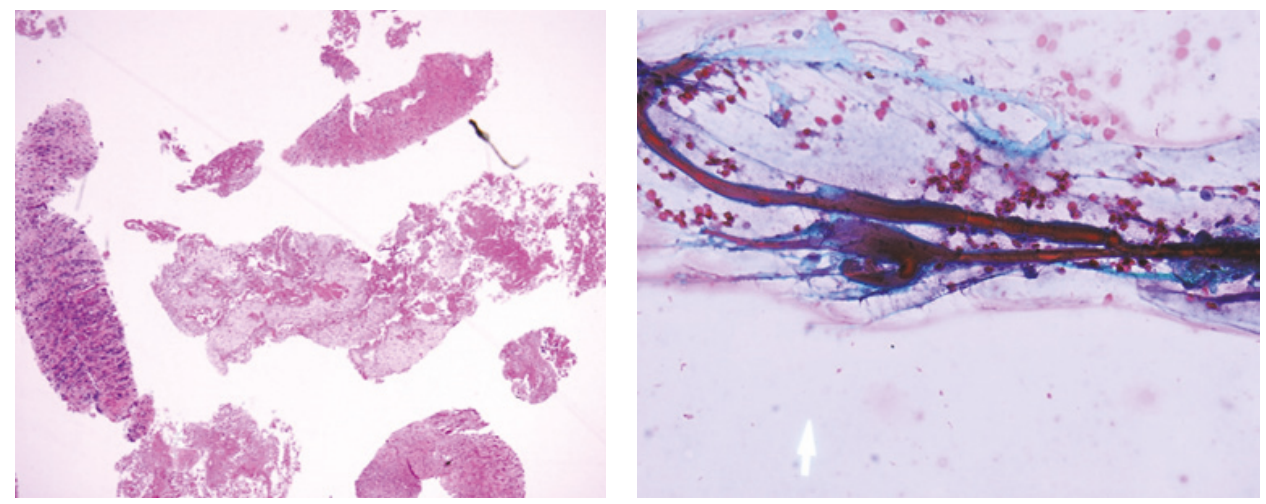

FIGURE 2: Fine needle aspiration revealed benign skeletal muscle, fibrous tissue, and few fragments of necrotic material.

\section{Conclusion}

While rare cases of $\mathrm{Q}$ fever presenting as inflammatory pseudotumor in the lung have been reported, we report the first case of $\mathrm{Q}$ fever presenting as a pseudotumor in the retroperitoneum and describe the major diagnostic challenges. Collectively, the histopathological findings for these pseudotumors associated with $C$. burnetii have not been specific, highlighting the need for heightened awareness of this rare presentation. Our case showed ischemic-like necrosis without inflammation or granulomas, which may be a unique finding. Recognition of this finding in correlation with serologic and molecular studies may help to provide accurate diagnosis in challenging cases of $C$. burnetii infection.

\section{Conflicts of Interest}

The authors declare that they have no conflicts of interest.

\section{References}

[1] M. Lee, J. J. Jang, Y. S. Kim et al., "Clinicopathologic features of Q fever patients with acute hepatitis," The Korean Journal of Pathology, vol. 46, no. 1, pp. 10-14, 2012.

[2] P. Parola et al., "Diagnosis of Q Fever," Journal of Clinical Microbiology, pp. 1823-1834, 1998.

[3] M. C. A. Wegdam-Blans, T. Vainas, M. R. Van Sambeek et al., "Vascular complications of Q-fever infections," European Journal of Vascular and Endovascular Surgery, vol. 42, no. 3, pp. 384-392, 2011.

[4] T. F. Hatchette and T. J. Marrie, "Atypical manifestations of chronic Q fever," Clinical Infectious Diseases, vol. 33, no. 8, pp. 1347-1351, 2001.

[5] D. T. Janigan and T. J. Marrie, "An inflammatory pseudotumor of the lung in Q fever pneumonia," The New England Journal of Medicine, vol. 308, no. 2, pp. 86-88, 1983.

[6] J. H. Lipton, T. C. Fong, M. J. Gill, K. Burgess, and P. D. Elliott, "Q fever inflammatory pseudotumor of the lung," Chest, vol. 92, no. 4, pp. 756-757, 1987.

[7] C. Sessa, L. Vokrri, P. Porcu, M. Maurin, J. P. Stahl, and J.L. Magne, "Abdominal aortic aneurysm and Coxiella burnetii infection: Report of three cases and review of the literature," Journal of Vascular Surgery, vol. 42, no. 1, pp. 153-158, 2005.

[8] M. E. O’Donnell, N. Manshani, C. McCaughey, C. Soong, and B. Lee, "Coxiella burnetii infection of an aortic graft with multiple vertebral body erosion," Journal of Vascular Surgery, vol. 45, no. 2, pp. 399-403, 2007.

[9] L. Senn, M. Franciolli, D. Raoult et al., "Coxiella burnetii vascular graft infection," BMC Infectious Diseases, vol. 5, no. 1, pp. 1-4, 2005.

[10] F. Baziaka, I. Karaiskos, L. Galani et al., "Large vessel vasculitis in a patient with acute Q-fever: A case report," IDCases, vol. 1, no. 3, pp. 56-59, 2014. 


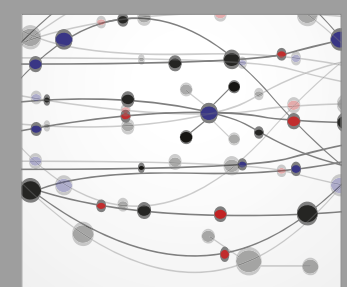

The Scientific World Journal
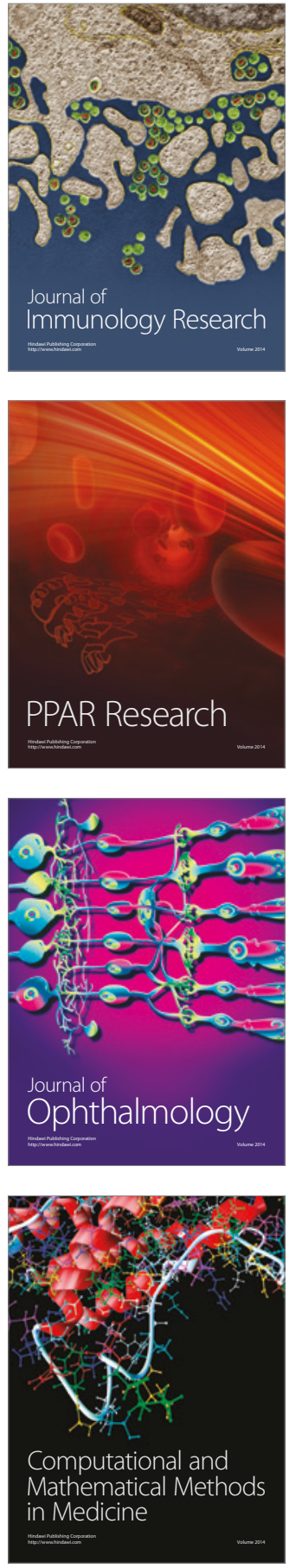

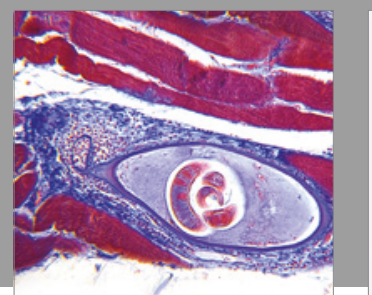

Gastroenterology Research and Practice
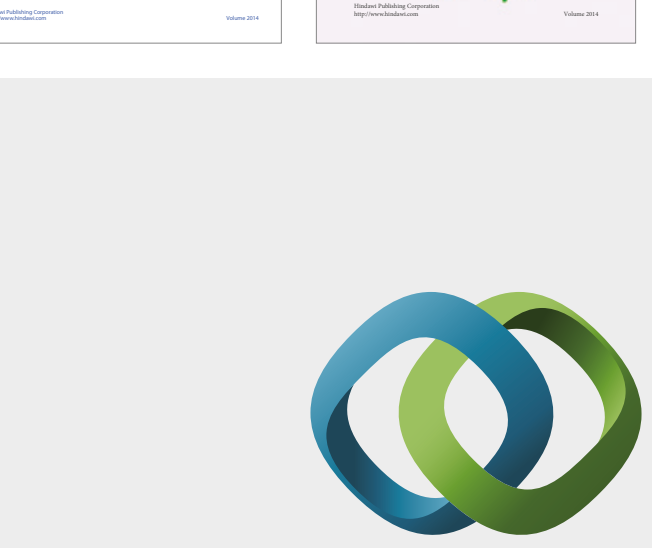

\section{Hindawi}

Submit your manuscripts at

https://www.hindawi.com
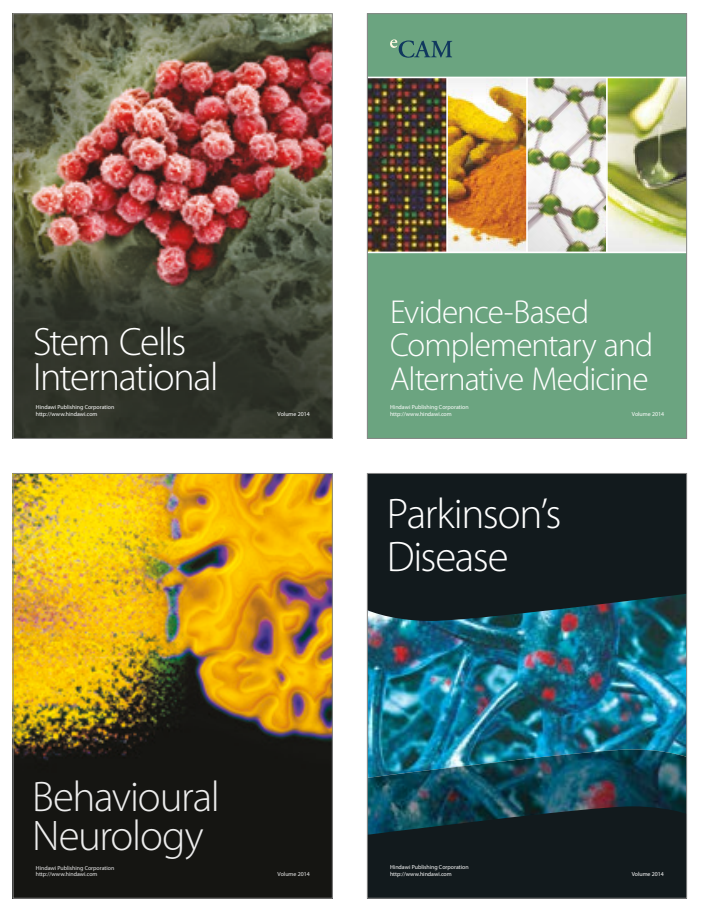
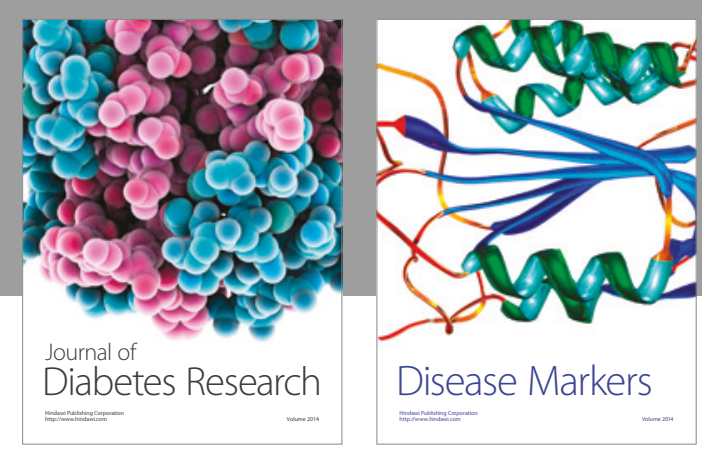

Disease Markers
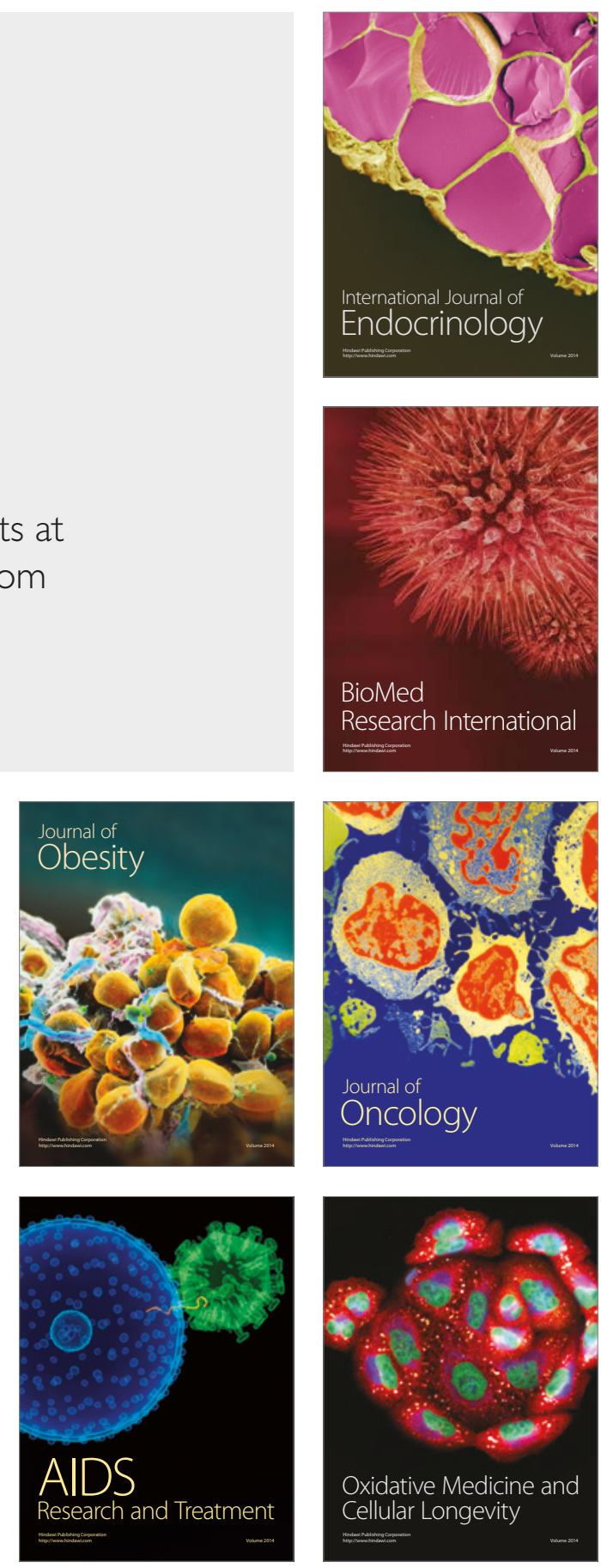